\title{
Must family/carers look after strangers? Post-DBS identity changes and related conflicts of interest
}

\section{Robin Mackenzie* \\ Kent Law School, Eliot College, University of Kent, Canterbury, Kent, UK \\ *Correspondence: r.mackenzie@kent.ac.uk}

Deep brain stimulation (DBS), a favored treatment option for Parkinson's disease and treatment resistant depression, restores disrupted brain mechanisms to default states and is likely to extend to mental and movement disorders and neurodegenerative conditions. All are associated with gradual cognitive, affective and/or behavioral changes. DBS confronts family/carers with emotionally, physically, and mentally trying challenges, as successful symptomatic relief may be accompanied by instantaneous apparent identity changes. Patients become restored to a previous state, "normal" or species-typical in ways they have never been, or placed in an enhanced state of subjective well-being. They are likely to feel like a new person, both to themselves and to others. How clinicians conceptualize patients' post-DBS personality changes has profound ethical implications not only for the patient but also for their family/carers. These amplify existing conflicts of interests.

Some involve patients, family/carers, and healthcare authorities. Family/carers looking after patients with neurodegenerative conditions, mood disorders, and brain injuries in the home conserve healthcare resources at considerable personal cost. They are increasingly subject to coercive expectations of affection and day-to-day care for the aging and infirm as demographic changes foster devolution of care from hospitals to homes. These resourcedriven policies tend to be couched in terms of the rhetoric of patient choice, e.g., deinstitutionalization of neurorehabilitation and hospice services on the grounds that most of us wish to die at home. Family/ carers are placed in an invidious position as they are expected to function almost as ancillary members of clinical teams in terms of providing care, yet lack the authority, training, remuneration, and legal safeguards accorded clinicians.

The potential of DBS to provide symptomatic relief could spare healthcare budgets and family/carers' personal resources.
Devolution from hospitals to homes depends upon family/carers providing dayto-day care, monitoring patients' conditions and taking momentous decisions, like assessing decision-making competence and capacity after the cognitive, affective, and behavioral changes DBS is likely to treat. As increasing proportions of the population will suffer from chronic conditions, or be involved in looking after the seriously impaired at home, the financial, and clinical implications of successful DBS treatment are immense. Patients, family/carers and society at large could reap significant benefits from symptomatic relief leading to returns to paid employment, increased social participation, and release from carer burden. Yet these benefits must be balanced against the risks of conflicts of interest arising from the impact of post-DBS personality changes on family/carers.

Both sudden and gradual personality/identity changes stress family/carers. Conditions like stroke and traumatic brain injury can cause sudden identity alterations, while gradual changes characterize neurodegenerative conditions. Family/carers experience poorer mental and physical health and more stress where patients' personalities have changed. They report more resilience when there has been cognitive but not affective change so affectionate relations are preserved. Caring for someone where love and affection continue is understandably easier than providing services for someone who may look the same, but feels and behaves like a stranger (Mackenzie and Sakel, 2011). Clinical strategies emphasize constructing narratives providing continuities of identity, as where neurorehabilitative goal-setting supports recovery of self and capacities after sudden changes, or narratives connecting past memories and present events preserve fading self-concepts in dementia. Family/carers who adhere to these stories are more able to continue to provide care on the basis of affection for those who still feel like their loved ones, despite clinically induced changes. Narratives provide a context of continuous meaning for patients' personality changes which allow for grief for the loss of capacities, but continuity of caring (Ylvisaker et al., 2008).

Post-DBS personality changes are different. Patients may not behave or feel like familiar damaged or diseased loved ones, but like healthy strangers with claims on family/carers' time, affection, and assets. While cognitive and behavioral incapacities characterize patients with stroke, brain injury, and dementia, affectionate mutuality often continues, so that family/carers feel that the relationship is maintained despite misfortune. Yet conditions where patients maintain their cognitive abilities, but lose their capacities for empathy, insight into their own behavior and considering others' interests, as in "acquired sociopathy" associated with behavioral variant frontotemporal dementia, are notorious for placing the maximum burden on carers (Mackenzie and Sakel, 2011). Thus, family/carers of DBS patients are likely to be faced with significant stresses associated with being linked to those who look like their loved ones, but may behave quite differently, value very different things, and be unconstrained by past ties of affection.

Family/carers are likely to feel guilty and conflicted if they prefer patients who were previously suffering from serious clinical symptoms, yet remained affectionate, to the same patients in their post-DBS state where DBS results in altered personalities, values, and choices over ways of life. Grieving for the lost person's presence may feel unethical in that serious symptoms have been alleviated, but, where the healthy person feels like a stranger, their claims on family/carers' time, affection, and assets may feel inappropriate and unjustified. Unanticipated breakdowns of relationships, dispersal of familial assets and inordinate stress on family/carers are likely outcomes, which should be addressed carefully in pre-DBS informed consent procedures. This is particularly 
crucial as competent patients whose values change post-DBS could repudiate advance decisions made pre-DBS.

Deep brain stimulation effects' reversibility also provokes unique ethical dilemmas. Neuromodulatory stimuli may be turned on or off and up or down. Using DBS to map connections between neural mechanisms, specific brain locations, subjective experiences and ways of behaving may provide evidence allowing for choice over where settings should be to ensure desired personality traits post-DBS. Accompanying disruptions are inevitable in diagnostic categories and conceptions of what constitutes normality, neurodiversity, and neurodysfunction. Clinicians may become able to use DBS to create tailor-made personalities for patients. After taxonomic upheavals, an increased range of personality traits are likely to become accepted as neurodiverse rather than neurodysfunctional. This all impacts on clinicians' ethical responsibilities, as it may be possible to provide DBS in ways which are clinically equivalent, but have varied outcomes in terms of patients' personality and behavioral changes. How choices amongst settings determining this should be made, and by whom, is unclear.

Patients are likely to choose settings enabling them to feel "better than well," whereas family/carers may prefer personality traits more like their familiar loved ones'. Should either be clinically preferable, it should prevail. Yet no ethical guidelines exist for choosing between clinically equivalent settings. Eschewing extreme settings may be deplored as coerced normalization and there are no clear grounds for considering specific settings as guaranteeing more authentic patient identities. Conflicts are inevitable over which settings should be chosen to enable which personality characteristics (Mackenzie, 2011). Boundaries between neurodiversity and neurodysfunction are likely to be bitterly contested.

Should symptomatic relief be accompanied by altered personality traits leading to relationship breakdown and dispersal of family assets, family/carers may want some say in choices over treatment outcomes. Yet as clinicians' duty of care is to patients alone, where post-DBS personality traits are clinically equivalent, patients should be accorded autonomous choice. Clinicians would retain a duty to ensure that patients were informed of the risks and options accompanying their choices. As patients' risk preferences are likely to alter with DBS treatment, questions arise over which of a range of possible states should be accepted as a basis for autonomous choices. Should the choice over settings of the patient in a risk-averse, risk-neutral, or risk-seeking state be accepted? As conditions treated by DBS involve cognitive, affective, and behavioral alterations, patients' pre-treatment states cannot be seen as more authentic than any of a range of clinically equivalent post-treatment states of being.

Clinicians may feel an ethical obligation to redress such conflicts of interest by including family/carers in the informed consent procedures, or suggesting they obtain legal advice on protecting familial assets pre-DBS. Yet clinicians also need to protect their patients. They should monitor refusals of DBS treatment and assess how autonomous patients' choices over post-DBS settings are in relation to undue influence or coercion. Family/carers may attempt to manipulate patients to refuse DBS or to choose settings which preserve affectionate bonds and family assets. Where patients eligible for DBS are incompetent, family/carers may be tempted to influence discussions over whether DBS is in patients' best interests, or to institutionalize them prematurely. Access to outside interference with DBS mechanisms and settings may need to be restricted, as may the range of settings able to be chosen.

This opinion has sketched out some conflicts of interest which may arise between healthcare authorities, clinicians, patients, and family/carers post-DBS treatment. Yet the promise of DBS to transform the well-being of all parties is commensurately immense. In the light of these factors, as well as others which space constraints prevent my mentioning, there is an urgent need for ethical guidelines on DBS.

\section{REFERENCES}

Mackenzie, R. (2011). Who should hold the remote for the new me? Cognitive, affective and behavioural side-effects of DBS and authentic choices over future personalities. AJOB Neurosci. 2, 18-19.

Mackenzie, R., and Sakel, M. (2011). "Neuroscience of cruelty as brain damage: legal framings of capacity and ethical issues in the neurorehabilitation of MND and bvFTD," in Law and Neuroscience, ed. M. Freeman (Oxford: Oxford University Press), 285-307.

Ylvisaker, M., Mcpherson, K., Kayes, N., and Pellett, E. (2008). Metaphoric identity mapping: facilitating goal setting and engagement in rehabilitation after traumatic brain injury. Neuropsychol. Rehabil. 18,713-741.

Received: 31 January 2011; accepted: 18 April 2011; published online: 09 May 2011.

Citation: Mackenzie R (2011) Must family/carers look after strangers? Post-DBS identity changes and related conflicts of interest. Front. Integr. Neurosci. 5:12. doi: 10.3389/ fnint.2011.00012

Copyright () 2011 Mackenzie. This is an open-access article subject to a non-exclusive license between the authors and Frontiers Media SA, which permits use, distribution and reproduction in other forums, provided the original authors and source are credited and other Frontiers conditions are complied with. 